\title{
O XENODIAGNÓSTICO E OS CRITÉRIOS PARA AVALIAR O NÍVEL DE PARASITEMIA DO PACIENTE CHAGÁSICO CRÔNICO
}

\author{
Elisabeth Bronfen e Nelson Junqueira Alvarenga
}

\begin{abstract}
A sensibilidade do xenodiagnóstico e sua relação com a parasitemia da fase crônica da doença de Chagas foi verificada em 104 pacientes crônicos. As ninfas das espécies de triatomíneos, simultaneamente utilizadas nos xenodiagnósticos destes pacientes, divididos em dois grupos, foram examinadas de maneiras diferentes. Os indices de positividade totais obtidos nos dois grupos $(40,0 \%$ e $42,4 \%)$ e os percentuais de positividade porpaciente sugerem uma reavaliafão do que se denomina a "quantificação da parasitemia do paciente chagásico crónico, considerando a metodologia de exame do xenodiagnóstico.
\end{abstract}

Palavras-chaves: Parasitemia. Doença de Chagas. Fase crônica. Xenodiagnóstico.

O xenodiagnöstico, junto com a hemocultura, apesar de ser o exame de certeza parasitológica para os indivíduos parasitados pelo Trypanosoma cruzi, apresenta baixa sensibilidade para a fase crônica da doença de Chagas. Esta sensibilidade tem sido explicada, principalmente, pela parasitemia escassa e irregular que caracteriza esta fase.

$O$ presente trabalho teve como objetivo reavaliar a sensibilidade do xenodiagnóstico através dos índices de positividade obtidos com a utilização de dois recursos metodológicos para o seu exame e a relação deles com o que se denomina a "quantificação da parasitemia do paciente chagásico crônico".

\section{MATERIAL E MÉTODOS}

Foram utilizados dois grupos de pacientes, procedentes do Estado de Minas Gerais, de maneira não simultânea. $O$ Grupo I, com 45 pacientes, pertencia a casuística do Laboratório de Biologia de Triatomineos e Epidemiologia da Doença de Chagas do Centro de Pesquisas "René Rachou", sendo 21 homens e 24 mulheres, com duas reações sorológicas concordantes para o Trypanosoma cruzi. O Grupo II, com 59 pacientes, sendo 49 homens e 10 mulheres, triados por sorologia anti- $T$. cruzi entre candidatos a doadores de sangue 1 . A faixa etária dos dois grupos compreendeu pacientes de 19 a 59 anos de idade.

O Grupo I foi submetido a um xenodiagnóstico com 90 insetos: 30 ninfas de $3{ }^{\circ}$ estádio de Panstrongylus

Centro de Pesquisas René Rachou FIOCRUZ, Belo Horizonte, MG.

Endereço para correspondência: Dra. Elisabeth Bronfen. Centro de Pesquisas René Rachou FIOCRUZ. CP: 1743, 30190 Belo Horizonte, MG.

Recebido para publicação em 05/09/90. megistus, Triatoma infestans e Rhodnius neglectus. O Grupo II, a um xenodiagnóstico com 120 insetos: 40 ninfas de $3^{\circ}$ estádio de $P$. megistus e $T$. infestans e 40 de 1. estádio de Dipetalogaster maximus.

Após a realização de cada xenodiagnóstico, verificava-se o número de ninfas engorgitadas e o daquelas que não haviam sugado, sendo estas desprezadas. As caixas contendo os triatomíneos alimentados eram mantidas em insetário climatizado a $27 \pm 1^{\circ} \mathrm{C}$ e $70,0 \%$ de umidade até o momento do exame. As ninfas dos dois grupos de xenodiagnósticos, encontradas mortas e endurecidas no dia do exame, não foram examinadas.

A dissecção das ninfas dos xenodiagnósticos do Grupo I era feita com a retirada de todo o tubo digestivo de cada inseto, que era acrescido de 5 microlitros de solução salina tamponada (PBS) e esmagado entre lâmina e lamínula.

As ninfas dos xenodiagnósticos do Grupo II eram colocadas em número de 10 por espécie de triatomineo em $5 \mathrm{ml}$ de solução esterilizante de WHITE durante 1 hora e 30 minutos. Esta esterilização prévia dos insetos permitia que as ninfas fossem examinadas em condições assépticas nas etapas seguintes, conduzidas em capela. De cada ninfa era retirado todo o conteúdo intestinal, com auxílio de duas pinças, constantemente flambadas, e colocado diretamente em tubo de ensaio com 500 microlitros de PBS estéril. Um total de 10 conteúdos intestinais por tubo de ensaio era triturado e homogeneizado com auxílio de bastão de vidro. Uma alíquota de 5 microlitros da suspensão era examinada entre lâmina e lamínula por 200 campos microscópicos de $400 \mathrm{X}$. Toda a suspensão negativa era semeada em tubo de ensaio contendo 2,5 $\mathrm{ml}$ de meio LIT com ampicilina. 
Bronfen E, Alvarenga NJ. Oxenodiagnóstico e os critérios para avaliar o nível de parasitemia do paciente chagásico crônico. Revista da Sociedade Brasileira de Medicina Tropical 24: 37-42, jan-mar, 1991

A esta semeadura do material intestinal do triatomineo, coletado e processado assepticamente, denominamos de xenocultura ${ }^{4}$. As xenoculturas eram incubadas a $28^{\circ} \mathrm{C}$ em estufa BOD-FANEM e examinadas após 20 dias.

$\mathrm{Na}$ análise estatistica dos dados foi utilizado o teste qui-quadrado, considerando o nível de significância para $\mathrm{p} \leq 0,05$.

\section{RESULTADOS}

A Tabela 1 mostra o número de insetos das três espécies utilizadas e examinadas nos dois grupos de xenodiagnósticos. O T. infestans foi a espécie com o maior número de ninfas que sugaram, nos dois grupos. A menor mortalidade observada foi do $R$. neglectus, no Grupo I e, para o $T$. infestans, no II. Estas duas espécies foram a de maior chance numérica de serem examinadas.

Tabela 1 - Capacidade alimentar e mortalidade das espécies de triatomineos simultaneamente utilizadas nos xenodiagnósticos dos pacientes chagásicos crônicos dos Grupos I e II

No de ninfas

\begin{tabular}{|c|c|c|c|c|c|c|c|c|}
\hline \multirow{2}{*}{$\begin{array}{l}\text { Espécie do } \\
\text { triatomineo }\end{array}$} & \multicolumn{2}{|c|}{ Aplicadas } & \multicolumn{2}{|c|}{ Sugaram (\%) } & \multicolumn{2}{|c|}{ Mortas (\%) } & \multicolumn{2}{|c|}{ Examinadas (\%) } \\
\hline & I & II & I & II & I & II & I & II \\
\hline P. megistus & 1350 & 2360 & $\begin{array}{c}1221 \\
(90,4)\end{array}$ & $\begin{array}{c}1545 \\
(65,5)\end{array}$ & $\begin{array}{c}93 \\
(7,6)\end{array}$ & $\begin{array}{c}165 \\
(10,7) \\
\end{array}$ & $\begin{array}{c}1128 \\
(83,5)\end{array}$ & $\begin{array}{r}1380 \\
(58,5) \\
\end{array}$ \\
\hline T. infestans & 1350 & 2360 & $\begin{array}{c}1312 \\
(97,2)\end{array}$ & $\begin{array}{c}2157 \\
(91,4)\end{array}$ & $\begin{array}{c}99 \\
(7,5)\end{array}$ & $\begin{array}{c}67 \\
(3,1) \\
\end{array}$ & $\begin{array}{c}1213 \\
(98,9)\end{array}$ & $\begin{array}{c}2090 \\
(88,6)\end{array}$ \\
\hline R. neglectus & 1350 & - & $\begin{array}{c}1286 \\
(95,3)\end{array}$ & - & $\begin{array}{c}58 \\
(4,5)\end{array}$ & - & $\begin{array}{c}1228 \\
(91,0)\end{array}$ & - \\
\hline D. maximus & - & 2360 & - & $\begin{array}{c}1326 \\
(56,2)\end{array}$ & - & $\begin{array}{c}166 \\
(12,5) \\
\end{array}$ & - & $\begin{array}{r}1160 \\
(49,2) \\
\end{array}$ \\
\hline Total & 4050 & 7080 & $\begin{array}{c}3819 \\
(94,3)\end{array}$ & $\begin{array}{c}5028 \\
(71,0)\end{array}$ & $\begin{array}{c}250 \\
(6,5)\end{array}$ & $\begin{array}{c}398 \\
(7,9)\end{array}$ & $\begin{array}{c}3569 \\
(88,1)\end{array}$ & $\begin{array}{c}4630 \\
(65,4)\end{array}$ \\
\hline
\end{tabular}

Dos 45 pacientes do Grupo I, $18(40,0 \%)$ foram parasitologicamente comprovados e, $25(42,4 \%)$ dos 59 pacientes do Grupo II. As Tabelas 2 e 3 mostram a positividade destes pacientes, agrupados pelo número de espécies e o total de ninfas e "pools" positivos.

A Tabela 4 mostra a positividade por espécie de triatomineo utilizada nos dois grupos de xenodiagnósticos. No Grupo I, o $P$. megistus positivou 10 pacientes (22,2\%); o $T$. infestans, $12(26,7 \%)$ e, o $R$. neglectus, 5 pacientes $(11,1 \%)$. No Grupo II, o $P$. megistus e o $T$. infestans positivaram 15 pacientes $(25,4 \%)$ e, o $D$. maximus, 13 (22,0\%).

Não houve diferença estatisticamente significativa entre a positividade das diferentes espécies de triatomíneos, na comprovação parasitológica dos 45 pacientes do Grupo I e dos 59 pacientes do Grupo II.

A Tabela 5 mostra a contribuição da xenocultura para a positividade de seis pacientes do Grupo II.

\section{DISCUSSÃO}

Os percentuais de positividade obtidos neste trabalho sugerem a utilização de mais de uma espécie de triatomíneo no xenodiagnóstico para a fase crônica da doença de Chagas, como foi mostrado na Tabela 4. Se os xenodiagnósticos dos dois grupos tivessem sido realizados só com o $P$. megistus, 18 pacientes positivos seriam considerados negativos ( 8 do Grupo I + 10 do Grupo II). Se só com o T. infestans, 16 (6 do Grupo I + 10 do Grupo II) e, se só com o $R$. neglectus ou o D. maximus, 25 ( 13 do Grupo I + 12 do Grupo II).

Freitas ${ }^{10}$ sugeria que a utilização de um maior número de ninfas por xenodiagnóstico aumentaria a chance de alguma delas se infectar. Entretanto, no presente trabalho, observou-se que o $R$. neglectus, com 1.228 ninfas examinadas (Tabela 1), não foi a espécie com maior positividade nos 45 xenodiagnósticos do Grupo I e o D. maximus, com 1.160 ninfas examinadas, a espécie com menor índice de positividade nos 59 xenodiagnósticos do Grupo II.

Se se pode excluir, destes resultados, a influência da susceptibilidade das espécies de triatomíneos ${ }^{9} \mathrm{e}$ o número de suas ninfas, na sensibilidade do xenodiagnóstico, qual seria, no presente trabalho, o envolvimento dos percentuais de positividade obtidos nos dois grupos, com a parasitemia escassa e irregular que 
Bronfen E, Alvarenga NJ. Oxenodiagnóstico e os critérios para avaliaro nível de parasitemia do paciente chagásico crônico. Revista da Sociedade Brasileira de Medicina Tropical 24: 37-42, jan-mar, 1991

Tabela 2 - Resultado da comprovação parasitológica de 18 pacientes chagásicos crônicos entre 45 submetidos ao xenodiagnóstico (Grupo I) agrupados pelo número de ninfas positivas por espécie de triatomineo.

\begin{tabular}{|c|c|c|c|c|c|}
\hline \multirow{2}{*}{$\begin{array}{l}\text { No de espécies } \\
\text { de triatomineos } \\
\text { positivas }\end{array}$} & \multirow{2}{*}{$\begin{array}{c}\text { Pacientes } \\
\text { (sexo-idade) }\end{array}$} & \multicolumn{4}{|c|}{$\begin{array}{c}\text { No de ninfas positivas/espécie/total de } \\
\text { examinadas (\% de positivas) }\end{array}$} \\
\hline & & P. megistus & T. infestans & R. neglectus & Subtotal \\
\hline \multirow{4}{*}{3} & LFTS (M-20) & $22 / 28(78,6)$ & $18 / 29(62,0)$ & $9 / 27(33,3)$ & $49 / 84(58,3)$ \\
\hline & WPS (M-26) & $5 / 28(17,8)$ & $8 / 27(29,6)$ & $2 / 26 \quad(7,7)$ & $15 / 81(18,5)$ \\
\hline & IK $(F-50)$ & $6 / 30(20,0)$ & $3 / 30(10,0)$ & $1 / 30 \quad(3,3)$ & $10 / 90(11,1)$ \\
\hline & Subtotal & $33 / 86(38,4)$ & $29 / 86(33,7)$ & $12 / 83(14,4)$ & $74 / 255(29,0)$ \\
\hline \multirow{4}{*}{2} & JTM (M-33) & $3 / 18(16,7)$ & $2 / 30 \quad(6,7)$ & $0 / 29(0)$ & $5 / 77 \quad(6,5)$ \\
\hline & $\mathrm{JCS}(\mathrm{M}-30)$ & $3 / 16(18,7)$ & $0 / 17(0)$ & $1 / 28 \quad(3,6)$ & $4 / 61(6,5)$ \\
\hline & MMRF (F-40) & $2 / 27(7,4)$ & $2 / 26(7,7)$ & $0 / 27(0)$ & $4 / 80(5,0)$ \\
\hline & Subtotal & $8 / 61(13,1)$ & $4 / 73(5,5)$ & $1 / 84(1,2)$ & $13 / 218(6,0)$ \\
\hline \multirow{5}{*}{1} & $\mathrm{AMCC}(\mathrm{F}-48)$ & $1 / 23(4,3)$ & $0 / 25(0)$ & $0 / 23(0)$ & $1 / 71(1,4)$ \\
\hline & OVL (M35) & $1 / 22(4,5)$ & $0 / 29(0)$ & $0 / 27(0)$ & $1 / 78(1,3)$ \\
\hline & $\mathrm{JDO}(\mathrm{M}-38)$ & $1 / 28(3,6)$ & $0 / 25(0)$ & $0 / 20(0)$ & $1 / 73(1,4)$ \\
\hline & MAS (M-36) & $3 / 23(13,0)$ & $0 / 30(0)$ & $0 / 30(0)$ & $3 / 83(3,6)$ \\
\hline & Subtotal & $6 / 96(6,2)$ & $0 / 109(0)$ & $0 / 100(0)$ & $6 / 305(2,0)$ \\
\hline \multirow{8}{*}{1} & JFG (M-39) & $0 / 20(0)$ & $1 / 23(4,3)$ & $0 / 25(0)$ & $1 / 68(1,5)$ \\
\hline & IPS (M-31) & $0 / 30(0)$ & $2 / 30(6,7)$ & $0 / 30(0)$ & $2 / 90(2,2)$ \\
\hline & PMS (M-30) & $0 / 28(0)$ & $1 / 25(4,0)$ & $0 / 23(0)$ & $1 / 76(1,3)$ \\
\hline & IMM (F-49) & $0 / 30(0)$ & $1 / 29(3,4)$ & $0 / 26(0)$ & $1 / 85(1,2)$ \\
\hline & BMM (M-39) & $0 / 30(0)$ & $1 / 25(4,0)$ & $0 / 20(0)$ & $1 / 75(1,3)$ \\
\hline & LB (M-35) & $0 / 30(0)$ & $2 / 30(6,7)$ & $0 / 28(0)$ & $2 / 88(2,3)$ \\
\hline & $\operatorname{CSP}(\mathrm{M}-35)$ & $0 / 23(0)$ & $1 / 28(3,6)$ & $0 / 30(0)$ & $1 / 81(1,2)$ \\
\hline & Subtotal & $0 / 191(0)$ & $9 / 190(4,7)$ & $0 / 182(0)$ & $9 / 563(1,6)$ \\
\hline \multirow[t]{3}{*}{1} & EFS (M-28) & $0 / 30(0)$ & $0 / 29(0)$ & $3 / 30(10,0)$ & $3 / 89(3,4)$ \\
\hline & Subtotal & $0 / 30(0)$ & $0 / 29(0)$ & $3 / 30(10,0)$ & $3 / 89(3,4)$ \\
\hline & Total & $47 / 464(10,1)$ & $42 / 487(8,6)$ & $16 / 479(3,3)$ & $105 / 1430(7,3)$ \\
\hline
\end{tabular}

caracteriza a fase crônica da doença de Chagas 8 ?

O xenodiagnóstico positivo na fase crônica da doença de Chagas é considerado um indicador que revelaria parasitemias baixas, intermediárias $\mathrm{e}$

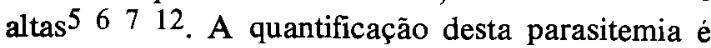
aferida através do número de xenodiagnósticos positivos por paciente, do número de ninfas positivas por xenodiagnóstico, do numero de caixas positivas por xenodiagnóstico por paciente ou do número de "pools" positivos por xenodiagnóstico.

No Grupo I, os 3 pacientes positivos com as três espécies apresentaram $11,1 \%, 18,5 \%$ e $58,3 \%$ de positividade; os 3, com duas espécies, $5,0 \%$ e $6,5 \%$, e, aqueles 12 , positivos com apenas uma espécie, de $1,2 \%$ a $3,6 \%$ de positividade. No Grupo II, 6 pacientes positivos com três espécies apresentaram $50,0 \%$ a $100 \%$ de positividade; 6 pacientes positivos com duas espécies, $25,0 \%$ a $44,4 \%$ e, 13 positivos com uma das espécies, uma positividade de $11,1 \%$ a $25,0 \%$. Portanto os maiores percentuais de positividade por paciente foram obtidos quando as ninfas foram examinadas em "pool" e não quando examinadas individualmente. Se estes percentuais podem estar refletindo a eficiência entre duas metodologias, elas sugerem uma avaliação da parasitemia do paciente chagásico crônico. Considerando os grupos de pacientes positivos com 3,2 ou uma espécie, observou-se percentuais muito diferentes. Eles podem ou não estarem aferindo parasitemias altas, intermediários ou baixas ou indicando que podemos obter maiores indices de positividade, usando recurso metodológico que os aumenta, por apresentar, inclusive, maior eficiência ${ }^{4}$.

A baixa densidade de flagelados implica uma grande dificuldade de visualização do parasita no intestino do triatomineo. Como já foi demonstra$\mathrm{do}^{3} 1314$, existem flutuações na positividade do triatomíneo infectado, especialmente visiveis através do exame pela compressão abdominal em diferentes dias após a infecção ${ }^{2}$. Considerando os niveis de adesão entre o $T$. cruzi e o epitélio intestinal do inseto ${ }^{15}$, a dissecção também esconde o parasita, presente em baixa quantidade. Desta maneira, a densidade de flagelados influi sobre a contagem final do número de insetos positivos por espécie de triatomineo e, mesmo, dentro da mesma espécie. Esta 
Bronfen E, Alvarenga NJ. Oxenodiagnóstico e os critérios para avaliar o nível de parasitemia do paciente chagásico crônico. Revista da Sociedade Brasileira de Medicina Tropical 24: 37-42, jan-mar, 1991

Tabela 3 - Resultado da comprovaçâo parasitológica de 25 pacientes chagásicos crônicos entre 59 indivíduos examinados (Grupo II) agrupados pelo número de "pools" positivos por espécie de triatomineo (examinados ao microscópio e após xenocultura).

\begin{tabular}{|c|c|c|c|c|c|}
\hline \multirow{2}{*}{$\begin{array}{c}\text { No de espécies } \\
\text { de triatomíneos } \\
\text { positivas }\end{array}$} & \multirow{2}{*}{$\begin{array}{c}\text { Pacientes } \\
\text { (sexo - idade) }\end{array}$} & \multicolumn{4}{|c|}{$\begin{array}{c}\text { No de "pools" positivos/espécie/total de } \\
\text { examinados (\% de positivos)* }\end{array}$} \\
\hline & & P. megistus & T. infestans & D. maximus & Subtotal \\
\hline \multirow{7}{*}{3} & NHR (F-30) & $2 / 2(100)$ & $4 / 4(100)$ & $2 / 2(100)$ & $8 / 8(100)$ \\
\hline & JAF $(\mathbf{M}-26)$ & $3 / 3(100)$ & $2 / 3(66,7)$ & $1 / 1(100)$ & $6 / 7(85,7)$ \\
\hline & JJS (M-37) & $3 / 3(100)$ & $2 / 4(50,0)$ & $2 / 2(100)$ & $7 / 9(77,8)$ \\
\hline & GMS (M-28) & $1 / 3(33,3)$ & $2 / 4(50,0)$ & $1 / 1(100)$ & $4 / 8(50,0)$ \\
\hline & DL (M-24) & $1 / 2(50,0)$ & $3 / 3(100)$ & $1 / 1(100)$ & $5 / 6(83,3)$ \\
\hline & RTL (F-42) & $2 / 2(100)$ & $1 / 3(33,3)$ & $3 / 3(100)$ & $6 / 8(75,0)$ \\
\hline & Subtotal & $12 / 15(80,0)$ & $14 / 21(66,7)$ & $10 / 10(100)$ & $36 / 46(78,3)$ \\
\hline \multirow{7}{*}{2} & EGB (M-49) & $0 / 3(0)$ & $2 / 4(50,0)$ & $2 / 2(100)$ & $4 / 9(44,4)$ \\
\hline & LFJ (F-19) & $0 / 3(0)$ & $1 / 3(33,3)$ & $1 / 2(50,0)$ & $2 / 8(25,0)$ \\
\hline & LRM (F-28) & $1 / 2(50,0)$ & $1 / 4(25,0)$ & $0 / 1(0)$ & $2 / 7(28,6)$ \\
\hline & JSM (M-42) & $1 / 3(33,3)$ & $0,4(0)$ & $1 / 1(100)$ & $2 / 8(25,0)$ \\
\hline & $\mathrm{DB}(\mathrm{M}-50)$ & $1 / 1(100)$ & $1 / 4(25,0)$ & $0 / 2(0)$ & $2 / 7(28,6)$ \\
\hline & JLB (M-47) & $1 / 2(50,0)$ & $0 / 4(0)$ & $1 / 1(100)$ & $2 / 7(28,6)$ \\
\hline & Subtotal & $4 / 14(28,6)$ & $5 / 23(21,7)$ & $5,9(55,5)$ & $14 / 46(30,4)$ \\
\hline \multirow{5}{*}{1} & LAT (M-24) & $1 / 2(50,0)$ & $0 / 4(0)$ & $0 / 2(0)$ & $1 / 8(12,5)$ \\
\hline & $\operatorname{RPS}(\mathrm{M}-46)$ & $1 / 3(33,3)$ & $0 / 4(0)$ & $0 / 1(0)$ & $1 / 8(12,5)$ \\
\hline & JBV (M-25) & $2 / 3(66,7)$ & $0 / 4(0)$ & $0 / 1(0)$ & $2 / 8(25,0)$ \\
\hline & $\operatorname{DSR}(M-33)$ & $2 / 3(66,7)$ & $0 / 4(0)$ & $0 / 1(0)$ & $2 / 8(25,0)$ \\
\hline & JCN (M-29) & $1 / 3(33,3)$ & $0 / 4(0)$ & $0 / 1(0)$ & $1 / 8(12,5)$ \\
\hline \multirow{7}{*}{1} & Subtotal & $7 / 14(50,0)$ & $0 / 20(0)$ & $0 / 6(0)$ & $7 / 40(17,5)$ \\
\hline & MA (M-33) & $0 / 2(0)$ & $1 / 3(33,3)$ & $0 / 2(0)$ & $1 / 7(14,3)$ \\
\hline & OJV (M-53) & $0 / 3(0)$ & $1 / 3(33,3)$ & $0 / 3(0)$ & $1 / 9(11,1)$ \\
\hline & LPS (M-38) & $0 / 3(0)$ & $1 / 3(33,3)$ & $0 / 1(0)$ & $1 / 7(14,3)$ \\
\hline & JCA (M-53) & $0 / 2(0)$ & $1 / 4(25,0)$ & $0 / 1(0)$ & $1 / 7(14,3)$ \\
\hline & WG (M-43) & $0 / 2(0)$ & $2 / 3(66,7)$ & $0 / 3(0)$ & $2 / 8(25,0)$ \\
\hline & Subtotal & $0 / 12(0)$ & $6 / 16(37,5)$ & $0 / 10(0)$ & $6 / 38(15,8)$ \\
\hline \multirow{5}{*}{1} & $\operatorname{MPD}(\mathrm{F}-31)$ & $0 / 3(0)$ & $0 / 4(0)$ & $2 / 3(66,7)$ & $2 / 10(20,0)$ \\
\hline & $\operatorname{MCC}(\mathrm{M}-20)$ & $0 / 2(0)$ & $0 / 3(0)$ & $1 / 3(33,3)$ & $1 / 8(12,5)$ \\
\hline & JL $(M-46)$ & $0 / 2(0)$ & $0 / 3(0)$ & $1 / 3(33,3)$ & $1 / 8(12,5)$ \\
\hline & Subtotal & $0 / 7(0)$ & $0 / 10(0)$ & $4 / 9(44,4)$ & $4 / 26(15,4)$ \\
\hline & Total & $23 / 62(37,0)$ & $25 / 90(27,8)$ & $19 / 44(43,2)$ & $67 / 196(34,2)$ \\
\hline
\end{tabular}

* Cada "pool" continha 10 conteúdos intestinais/espécie de triatomíneó.

Tabela 4-Positividáde dos xenodiagnósticos por espécie de triatomíneo obtida no total dos dois grupos (I e II) de pacientes chagásicos crônicos e entre os indivíduos positivos por grupos.

\begin{tabular}{|c|c|c|c|c|}
\hline \multirow[b]{2}{*}{$\begin{array}{l}\text { Espécie de } \\
\text { triatomineo }\end{array}$} & \multicolumn{2}{|c|}{$\begin{array}{l}\text { No de pacientes positivos/ } \\
\text { /total de estudados } \\
\text { (\% positivos) }\end{array}$} & \multicolumn{2}{|c|}{$\begin{array}{l}\% \text { de positividade na } \\
\text { amostra positiva } \\
\text { por grupo }\end{array}$} \\
\hline & $\begin{array}{l}\text { Grupo I } \\
n=45\end{array}$ & $\begin{array}{c}\text { Grupo II } \\
n=59\end{array}$ & $\begin{array}{l}\text { Grupo I } \\
n=18\end{array}$ & $\begin{array}{c}\text { Grupo II } \\
n=25\end{array}$ \\
\hline P. megistus & $10(22,2)$ & $15(25,4)$ & 55,5 & 60,0 \\
\hline T. infestans & $12(26,7)$ & $15(25,4)$ & 66,7 & 60,0 \\
\hline R. neglectus & $5(11,1)$ & NR & 27,3 & NR \\
\hline D. maximus & $\mathbf{N R}^{*}$ & $13(22,0)$ & NR & 52,0 \\
\hline
\end{tabular}

*NR = Nāo realizado.

Grupo I: $\mathrm{X}^{2}=2,63$, g. $1 .=2$

Grupo II: $X^{2}=2,58$, g. $1 .=2$ 
Bronfen E, Alvarenga NJ. Oxenodiagnóstico e os critérios para avaliar o nível de parasitemia do paciente chagásico crônico. Revista da Sociedade Brasileira de Medicina Tropical 24: 37-42, jan-mar, 1991

Tabela 5 - Contribuiçāo da xenocultura para a positividade de "pools" do P. megistus (P.m.), T. infestans (T.i.) e D. maximus (D.m.) negativos no exame ao microscópio dos xenodiagnósticos de seis pacientes do Grupo II.

\begin{tabular}{|c|c|c|c|c|c|c|c|c|c|c|c|c|c|c|c|c|c|c|}
\hline \multirow{2}{*}{$\begin{array}{c}\text { Pacientes } \\
\text { Espécie do triatonineo }\end{array}$} & \multicolumn{3}{|c|}{ NHR } & \multicolumn{3}{|c|}{ JAF } & \multicolumn{3}{|c|}{ RTL } & \multicolumn{3}{|c|}{ EGB } & \multicolumn{3}{|c|}{ LFJ } & \multicolumn{3}{|c|}{ LPS } \\
\hline & P.m & I.i & $D \cdot m$ & $P . m$ & $T . i$ & D.t. & P.IB & $T . i$ & D.m & P.m & T.i & D.m & P.m & $T . j$ & D.m & $P, m$ & Ti & $D_{m}$ \\
\hline $\begin{array}{l}\text { T. tolal de "pools" examinados } \\
\text { espécie de triatomineo }\end{array}$ & 2 & 4 & 2 & 3 & 3 & 1 & 2 & 3 & 3 & 3 & 4 & 2 & 3 & 3 & 2 & 3 & 3 & 1 \\
\hline $\begin{array}{l}\text { X: de "poots" positivoslespécie } \\
\text { de Inalomineo no exame ao } \\
\text { microscupio }\end{array}$ & $\begin{array}{c}2 / 2 \\
(100 \%)\end{array}$ & $\begin{array}{l}3 / 4 \\
(75 \%)\end{array}$ & $\begin{array}{c}2 / 2 \\
(100 \%)\end{array}$ & $\begin{array}{c}2,3 \\
(66,7 \%)\end{array}$ & $\begin{array}{c}1 / 3 \\
(33,3 \%)\end{array}$ & 111 & $\begin{array}{c}2 / 2 \\
(100 \%)\end{array}$ & $\begin{array}{l}0 B \\
(0 \%)\end{array}$ & $\begin{array}{c}3 / 3 \\
(100 \%)\end{array}$ & $\begin{array}{l}03 \\
(0 \%)\end{array}$ & $\begin{array}{c}1 / 4 \\
(25,0 \%)\end{array}$ & $\begin{array}{c}2 / 2 \\
(100 \%)\end{array}$ & $\begin{array}{l}0 / 3 \\
0 \%)\end{array}$ & $\begin{array}{c}13 \\
(33,3 \%)\end{array}$ & $\begin{array}{l}0 / 2 \\
(0 \%)\end{array}$ & $\begin{array}{l}03 \\
(0 \%)\end{array}$ & $\begin{array}{l}013 \\
(0 \%)\end{array}$ & $\begin{array}{l}\text { W1 } \\
10 \%)\end{array}$ \\
\hline $\begin{array}{l}\text { У. total de "pools" positivos } \\
\text { no exame ao microscópio }\end{array}$ & & $\begin{array}{c}718 \\
(87,5 \%) \\
\end{array}$ & & & $\begin{array}{c}47 \\
(57,1 \%)\end{array}$ & & & $\begin{array}{c}5 / 8 \\
(62.5 \%) \\
\end{array}$ & & & $\begin{array}{c}3 / 9 \\
(33,3 \%)\end{array}$ & & & $\begin{array}{c}1 / 8 \\
(12,5 \%)\end{array}$ & & & $\begin{array}{c}077 \\
(0 \%]\end{array}$ & \\
\hline $\begin{array}{l}\text { V. de "pools" positivos/especie } \\
\text { de triatomine a apos renocultura }\end{array}$ & - & $1 / 1$ & - & $1 / 1$ & $1 / 2$ & - & - & $1 / 3$ & - & $0 / 3$ & $1 / 3$ & - & $0 / 3$ & $0 / 2$ & $1 / 2$ & $\alpha / 3$ & $1 / 3$ & $0: 1$ \\
\hline $\begin{array}{l}\text { Vi tolal de "pools" positivos após } \\
\text { xenocultura }\end{array}$ & & $\begin{array}{c}8 / 8 \\
(100 \%)\end{array}$ & & & $\begin{array}{c}6 / 7 \\
(85,7 \%)\end{array}$ & & & $\begin{array}{c}688 \\
(75,0 \%)\end{array}$ & & & $\begin{array}{c}4 / 9 \\
(44,4 \%)\end{array}$ & & & $\begin{array}{c}2 \% \\
(25,0 \%) \\
\end{array}$ & & & $\begin{array}{c}177 \\
(14,3 \%)\end{array}$ & \\
\hline $\begin{array}{l}\text { N. total de "pools" positivos } \\
\text { especie de triatomineo }\end{array}$ & $\begin{array}{c}22 \\
(100 \%)\end{array}$ & $\begin{array}{c}4 / 4 \\
(100 \%)\end{array}$ & $\begin{array}{c}2 / 2 \\
(100 \%)\end{array}$ & $\begin{array}{c}3 / 3 \\
(100 \%)\end{array}$ & $\begin{array}{c}2 / 3 \\
(66,7 \%)\end{array}$ & $\begin{array}{c}111 \\
(100 \%)\end{array}$ & $\begin{array}{c}2 / 2 \\
(100 \%)\end{array}$ & $\begin{array}{c}13 \\
(33,3 \%)\end{array}$ & $\begin{array}{c}3 / 3 \\
(100 \%)\end{array}$ & $\begin{array}{c}0 / 3 \\
(0 \%)\end{array}$ & $\begin{array}{c}2 / 4 \\
(50,0 \%)\end{array}$ & $\begin{array}{c}2 / 2 \\
(100 \%)\end{array}$ & $\begin{array}{c}0 / 3 \\
(0 \%)\end{array}$ & $\begin{array}{c}113 \\
(33,3 \%)\end{array}$ & $\begin{array}{c}1 / 2 \\
(50,0 \%)\end{array}$ & $\begin{array}{c}0 / 3 \\
\langle 0 \%)\end{array}$ & $\begin{array}{c}1 / 3 \\
(33.3 \%)\end{array}$ & $\begin{array}{r}01 \\
\langle 0 \%) \\
\end{array}$ \\
\hline
\end{tabular}

influência é interpretada como susceptibilidades diferentes interespécies ou individuais dentro de uma mesma espécie 11 .

A xenocultura, amplificando em meio LIT, as interações triatomíneo- $T$. cruzi de baixa densidade de flagelados negativos ao exame microscópico convencional, mostrou também um outro aspecto a ser considerado na quantificação da parasitemia do paciente chagásico crônico.

As Tabelas 3 e 5 mostraram como seis, dos 25 pacientes positivos do Grupo II, aumentaram suas positividades após a xenocultura. O paciente NHR, sem mais um "pool" positivo, mostraria $87,5 \%$ de positividade (7tubos positivos em 8 examinados ao microscópio) (Tabela 5) e não $100 \%(8 / 8)$ (Tabela 3). $O$ paciente JAF teria mostrado uma positividade de $57,1 \%(4 / 7)$ e não $85,7 \%(6 / 7)$; RTL, $62,5 \%(5 / 8)$; EGB, $33,3 \%$; LFJ, $12,5 \%$ e o paciente LPS não teria sido parasitologicamente comprovado e sua positividade não teria sido $14,3 \%(1 / 7)$.

Os resultados do presente trabalho sugerem fortemente que se reavalie o conceito de "quantificação de parasitemia do paciente chagásico crônico" uma vez ter-se demonstrado que modificações na metodologia de exame do xenodiagnóstico alteram seus indices de positividade individual.

\section{$S U M M A R Y$}

Xenodiagnosis sensibility and its relation to Chagas' disease chronic phase parasitemia was verified in 104 patients. The nymphs of the triatomine species, simultaneously utilized in the xenodiagnosis of these patients, divided into two grupos, were examined in different ways. The positivity rates achieved in the two groups $(40,0 \%$ and
$42,4 \%)$ and the individual positivity rates suggest a reavaliation in what is called "quantification of the chronic chagasic patient parasitemia", considering the methodology used in the xenodiagnosis examinations.

Key-words: Parasitemia. Chagas' disease. Chronic phase. Xenodiagnosis.

\section{REFERÊNCIAS BIBLIOGRÁFICAS}

1. Bronfen E, Chiari E. Potencial de risco da transmissāo transfusional da doença de Chagas em Belo Horizonte (MG). Revista da Sociedade Brasileira de Medicina Tropical 21: 29-32, 1988.

2. Bronfen E, Dias JCP, Gouveia SC. Infecção experimental de Triatoma infestans e Panstrongylus megistus pela cepa Y do Trypanosoma cruzi (Silva e Nussenzweig, 1953). Revista de Patologia Tropical 13: 1-7, 1984.

3. Bronfen E, Rocha FSA, Dias JCP. Quantitative studies of Trypanosoma cruzi transmission. In: Resumos da VIII Reuniāo Anual sobre Pesquisa Básica em Doença de Chagas, Caxambu, pág. 63, 1981.

4. Bronfen E, Rocha FSA, Machado GBN, Perillo MM, Romanha AJ, Chiari E. Isolamento de amostras do Trypanosoma cruzi por xenodiagnóstico e hemocultura de pacientes na fase crônica da Doença de Chagas. Memórias do Instituto Oswaldo Cruz 84: 237-240, 1989.

5. Cançado JR Tratamento Específico. In: Cançado JR, Chuster M, (ed.) Cardiopatia Chagásica. Fundação Carlos Chagas, Belo Horizonte p. 327-355, 1985.

6. Castro CN. Influência da parasitemia no quadro clínico da Doença de Chagas, Tese de Mestrado, Universidade de Brasilia, Brasília, DF, 1978.

7. Cerisola JA, Rohwedder RW, Segura E, Del Prado CE, Alvarez M, Martini GW. El xenodiagnostico. In: International Symposium On New Approaches In American 
Bronfen E, Alvarenga NJ. Oxenodiagnóstico e os critérios para avaliar o nível de parasitemia do paciente chagásico crônico. Revista da Sociedade Brasileira de Medicina Tropical 24: 37-42, jan-mar, 1991

Trypanosomiasis Research, Pan American Health Organization, Belo Horizonte. Session III. B.4., 1975.

8. Chagas C. Nova tripanozomiaze humana. Estudos sobre a morfolojia e o ciclo evolutivo do Schizotrypanum cruzi n. gen. n. sp., ajente etiolojico de nova entidade morbida do homem. Memórias do Instituto Oswaldo Cruz 1: 159 218, 1909.

9. Dias E. Xenodiagnósticos seriados em cães infectados com amostras venezuelanas de "Schizotrypanum cruzi". Brasil-Médico 54: 859-861, 1940.

10. Freitas JLP. Contribuição para o estudo do diagnóstico da moléstia de Chagas por processos de laboratório. Tese de Doutorado. Universidade de São Paulo, São Paulo, SP, 1947.

11. Maudlin I. Inheritance of susceptibility of Trypanosoma cruzi infection in Rhodnius prolixus. Nature 262: 214$215,1976$.

12. Pereira JB, Willcox HPF, Marcondes CB, Coura JR
Parasitemia em pacientes chagásicos crônicos avaliada pelo índice de triatomíneos infectados no xenodiagnóstico. Revista da Sociedade Brasileira de Medicina Tropical 22: 39-44, 1989.

13. Phillips NR. Experimental studies on the quantitative transmission of Trypanosoma cruzi: aspects of the rearing, maintenance and testing of vector material, and of the origin and course of infection in the vector. Annals of Tropical Medicine and Parasitology 54: 397-414, 1960.

14. Schenone H, Rojo M, Rojas A, Concha L. Positividad diurna y nocturna del xenodiagnóstico en un paciente con infección chagásica crónica de parasitemia permanente. Boletin Chileno de Parasitologia 32: 63-66, 1977.

15. Zeledón R, Bolaños R, Rojas M. Scanning electron microscopy of the final phase of the life cycle of Trypanosoma cruz $i$ in the insect vector. Acta Tropica 41: 39 $43,1984$. 